N. E. Piskunov, W. W. Weiss, D. F. Gray, eds.

\title{
A Few Things We Do Not Know About Stars and Model Atmospheres
}

\author{
Robert L. Kurucz \\ Harvard-Smithsonian Center for Astrophysics \\ 60 Garden Street, Cambridge, MA 02138, USA
}

\begin{abstract}
A similar presentation has been published in the volume New Quests in Stellar Astrophysics: The Link between Stars and Cosmology Astrophysics and Space Science Library, vol. 274 (eds. M. Chavez, A. Bressan, A. Buzzoni, and D. Mayya) Dordrecht: Kluwer, pp. 1-10, 2002. Here I quote the abstract and list only the section headings.
\end{abstract}

Keywords: model atmospheres, convection, microturbulent velocity, spectroscopy, solar spectra, stellar spectra, abundances, solar abundances, atomic line data, molecular line data, Cepheids.

We list a few things that we do not understand about stars and that most people ignore. All of these are very hard problems. We can learn more about cosmology by working on the reduction of systematic errors introduced by the problems listed below than by trying to derive cosmological results that are highly uncertain.

1. Optimism and pessimism.

2. We do not know how to make realistic model atmospheres.

3. We do not understand convection.

4. We do not consider the variation in microturbulent velocity.

5. We do not understand spectroscopy.

6. We do not have good spectra of the sun or any other star.

7. We do not have energy distributions for the sun or any other star.

8. We do not know how to determine abundances.

9. We do not know the abundances of the sun or any other star.

10. We do not have good atomic and molecular data.

11. One half the lines in the solar spectrum are not identified.

12. Cepheids have convective pulsation but the models do not.

13. We do not have high quality spectra over a full cycle for any Cepheid. 
14. We do not understand abundance evolution in early type stars.

15. Many early type stars are oblate fast rotators.

Also see my paper Atomic and molecular data needs for astrophysics, presented at The 3rd International Conference on Atomic and Molecular Data, Gatlinburg, Tennessee, April 24-27, 2002. to be published by AIP Conference Proceedings, ed. D.R. Schultz, 2002. The punchline there is:

We can produce more science by investing in laboratory spectroscopy rather than by building giant telescopes that collect masses of data that cannot be correctly interpreted. 